\title{
Situación de las Emergencias Pediátricas en tiempos de Covid19
}

\section{The State of Pediatric Emergencies in times of Covid19}

\author{
Viviana Pavlicich ${ }^{1}$
}

Durante los primeros meses del año 2020 la pandemia de SARS CoV2 cambio la forma de vida de las personas. En lo que a servicios de salud se refiere, estos sufrieron una transformación organizacional para responder con la mayor seguridad posible a la atención de los pacientes, intentando disminuir el riesgo de contagio entre ellos y al personal sanitario. Los Servicios de Emergencia, convertidos en el lugar del primer impacto una vez suspendida la atención primaria y los consultorios médicos, debieron adaptarse a la nueva situación epidemiológica.

El comportamiento diferente de la nueva enfermedad en los niños respecto a los adultos obligó a pensar en una preparación y abordaje con algunas diferencias entre los Servicios de Emergencia Pediátricos (SEP) y aquellos que atenderían a pacientes adultos.

Series de casos publicadas sobre pacientes pediátricos procedentes de China, Italia y EE. UU. proporcionaron datos clínicos y clarificaron los síndromes de presentación de la Covid-19 en niños ${ }^{(1)}$. La tranquilidad impartida por información respecto a la moderada prevalencia de casos asintomáticos u oligosintomatico y la baja tasa de hospitalización en pediatría fue alterada por la aparición del Síndrome de inflamación multisistémica semanas después de haberse detectado la infección, o en niños negativos en quienes no se conocía su estado de inmunidad respecto a la nueva enfermedad ${ }^{(2)}$.
A tres meses de declarada la pandemia, los SEP ya no son los de antes. Se han instaurado flujos diferenciados de pacientes, en algunos casos respiratorios y no respiratorios, y en otros se ha ampliado la clasificación, separándolos en Sospecha de Covid-19 y No Covid-19. Se han implementado medidas de protección personal y se han realizado intensas capacitaciones en el uso adecuado de las mismas.

Lograr el cambio de conductas profesionales, personales y sociales dentro de las instituciones sanitarias fue un verdadero desafío. Sin embargo, la motivación en el aprendizaje, que partió de la preocupación de contraer o trasmitir la enfermedad, posibilitó que estos cambios sucedan en un corto tiempo.

Implementamos métodos nuevos de intubar, reanimar, ventilar y trasladar al paciente. La transmisión comunitaria declaraba: cualquiera puede ser Covid-19 positivo, y la demora del resultado de la prueba ante medidas que no pueden demorarse, implicó evitar la aerosolización y el contacto con secreciones en todos los pacientes que requirieron manejo de la vía aérea.

Otro desafío que vale la pena mencionar, fue el de enfrentarnos con nuestras viejas infraestructuras hospitalarias. Ellas denotan un concepto de arquitectura sanitaria que no privilegia la prevención de la transmisión de infecciones ni la protección de la privacidad del enfermo. Los pabellones sanitarios

\footnotetext{
${ }^{1}$ Hospital General Pediátrico Niños de Acosta Ñu. Departamento de Urgencias. San Lorenzo, Paraguay.

Correspondencia: Viviana Pavlicich.Correo:p_viviana@hotmail.com

Recibido: 13/06/20 Aceptado:19/06/2020

Doi: https://doi.org/10.31698/ped.47022020003
}

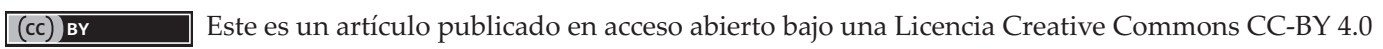


antiguos reducidos a habitaciones o salas para 5 o 6 pacientes siguen el mismo patrón que atenta contra la intimidad, el contagio, e impide que un niño hospitalizado pueda estar con sus dos padres en lugar con solo uno de ellos.

Mientras los casos confirmados de Covid 19 se acumulaban y nos adentrábamos en el invierno, pudimos observar algunos cambios. Las más de 12.000 consultas en nuestro servicio durante el mes de mayo en los años 2018 y 2019, se redujeron en un $80 \%$. (Fig 1) Si bien la campaña "Quédate en casa" y el temor al contagio puede haber influido en este descenso, estimo que la disminución de la carga de enfermedad debido a la falta de transmisión viral por el confinamiento también cumplió un rol importante.

La magnitud de este descenso sugiere que una combinación de factores biológicos, psicológicos y sociales influye en las decisiones de las familias de asistir a los Servicios de Urgencia y Emergencia.

Al finalizar mayo observamos una disminución de la hospitalización desde el SEP del $50 \%$, pero con un aumento relativo al número de consultas (Fig 2). Acudieron menos pacientes, pero con más necesidad de atención urgente. Esto lo corroboramos con el aumento de un $7 \%$ en los niveles I, II y III de prioridad del triage.

Entre ellas tuvimos que lamentar un incremento del número de peritonitis y de sepsis de origen intrabdominal como consecuencia de las consultas tardías. No está claro el motivo de esta demora. Existe bibliografía que postula que las consultas tardías se produjeron por temor de la población a acercarse a los hospitales. Sin embargo, en nuestro caso, que estos niños hayan consultado previamente parece indicar que podría tratarse de una organización confusa de la red sanitaria en lo que se refiere a pacientes pediátricos quirúrgicos agravada por la reorganización de los servicios durante el inicio de la pandemia ${ }^{(3)}$.

La carga de enfermedad respiratoria en mayo, (bronquiolitis, laringitis, neumonías, crisis asmáticas, síndromes bronquiales obstructivos recurrentes, enfermedad tipo influenza e infecciones de las vías respiratorias), presentó una disminución del $97 \%$ respecto a los dos años anteriores en nuestro servicio. Estos datos obtenidos de nuestro registro informático de diagnósticos de la consulta en urgencia, demostró una disminución de 5800 pacientes atendidos en un mes. (Fig. 3 y Fig. 4 )

Aunque la pronunciada disminución de enfermedades respiratorias puede explicarse por las medidas extremas de confinamiento, creo necesario evaluar las intervenciones que serán convenientes implementar de aquí en adelante en las escuelas para disminuir la trasmisión de los virus respiratorios durante el invierno.

Aprender sobre estos cambios en la asistencia puede ofrecer una oportunidad para encontrar formas alternativas de apoyar a las familias fuera del los SEP en el futuro ${ }^{(4)}$.

La disminución de las consultas permitió dedicar más tiempo a pacientes más complejos, lo que mejoró la calidad de la atención y la satisfacción de los profesionales. El primer aspecto no es fácilmente demostrable sin mediciones específicas, pero, con respecto al segundo, el personal sanitario manifestó esta sensación en reiteradas oportunidades.

Los niños con frecuencia se presentan a los SEP con enfermedades que no requieren atención de emergencia. Esto puede deberse a la ansiedad de los padres o a la dificultad para acceder a los servicios médicos de la comunidad. No debemos olvidar otra lección que aprendimos durante esta pandemia: es posible un mejor uso del departamento de emergencias $^{(5)}$.

Otro cambio notable fue el crecimiento exponencial de las capacitaciones a distancia y las consultas a través de telemedicina. Las reuniones de servicio, ateneos bibliográficos y clínicos aumentaron su frecuencia y fueron más concurridos. La comunicación dentro del equipo mejoró, así como la integración con los profesionales de enfermería gracias a la creación de canales de comunicación específicos a diferentes funciones o procesos.

Los niños enfermos o heridos y sus familias tienen necesidades únicas que pueden magnificarse cuando la dolencia del niño es grave o pone en 
peligro la vida. La disponibilidad de recursos y la preparación pediátrica en los Servicios de Urgencia y Emergencia es una necesidad. Brindar atención urgente de alta calidad requiere una infraestructura diseñada para apoyar la atención de los pacientes pediátricos y sus familias. Por lo tanto, es importante que los médicos, administradores y personal de apoyo colaboren con expertos en cuidados agudos pediátricos para optimizar la atención y así minimizar la morbilidad y mortalidad en niños como resultado de enfermedades y lesiones ${ }^{(6)}$.

\section{REFERENCIAS}

1. Zachariah P. Epidemiology, Clinical Features, and Disease Severity in Patients with Coronavirus Disease 2019 (COVID-19) in a Children's Hospital in New York City, New York. JAMA Pediatric. 2020; Published online June 3, doi: http://dx.doi.org/10.1001/amapediatrics.2020.2430

2. Verdoni L, Mazza A, Gervasoni A, Martelli L, Ruggeri M, Ciuffreda M, et al. An outbreak of severe Kawasaki-like disease at the Italian epicentre of the SARS-CoV-2 epidemic: an observational cohort study [Internet]. The Lancet 2020[citado el 05 jun 2020];395(10239): 1771-1778. doi: http://dx.doi.org/10.1016/S0140-6736(20)31103-X

3. Snapiri O, Rosenberg Danziger C, Krause I, Kravarusic D, Yulevich A, Balla U, et al. Delayed diagnosis of paediatric appendicitis during the COVID-19 pandemic [Internet]. Acta Paediatr. 2020[citado el 02 jun 2020];10.1111/apa.15376. doi: http://dx.doi.org/10.1111/apa.15376
Esta situación epidemiológica nos obligó a hacer cambios y se produjeron inversiones a nivel sanitario. Es necesario, entonces, elaborar un modelo de atención de las emergencias pediátricas que no sea igual al periodo prepandémico. Es una oportunidad para que las mejoras en triage, infraestructura, educación sanitaria a la población, aplicación de la telemedicina, representen avances que se sostengan en el tiempo.

4. Dann L, Fitzsimons J, Gorman k, Hourihane J, Okafor I. Disappearing act: COVID-19 and paediatric emergency department attendances [Internet]. Arch Dis Child. 2020 Epub ahead of print [citado el 08 jun 2020]; doi: http://dx.doi.org/10.1136/archdischild-2020-319654

5. Pata D, Gatto A, Buonsenso A, Chiaretti A. A COVID-19 outbreak's lesson: best use of the pediatric emergency department [Internet]. Acta Paediatr. 2020[citado el 12 jun 2020]. doi:http://dx.doi.org/10.1111/APA.15386

6. Moore B, Shah MI, Owusu-Ansah S, Gross T, Brown K, Gausche-Hill M, et al. Pediatric Readiness in Emergency Medical Services Systems [Internet]. Pediatrics. 2020[citado el 08 jun 2020];145(1)e20193307. doi: http://dx.doi.org/10.1542/peds.2019-3307

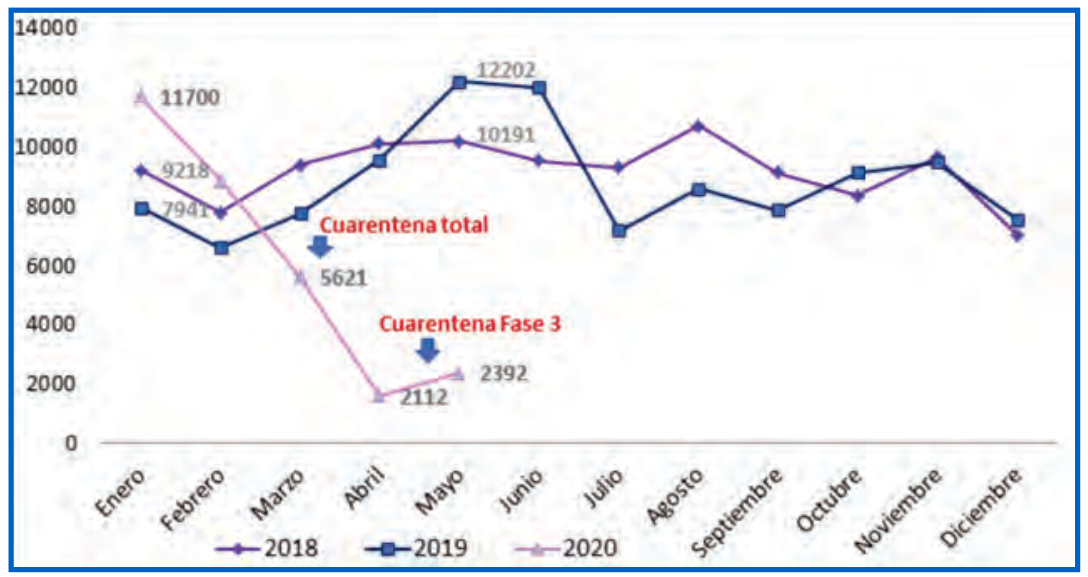

Fig 1. Consultas en el servicio de emergencias pediátricas, años 2018, 2019 y periodo pandemia hasta mayo 2020. Hospital General Pediatrico Niños de Acosta Nu. 
Pavlicich $V$.

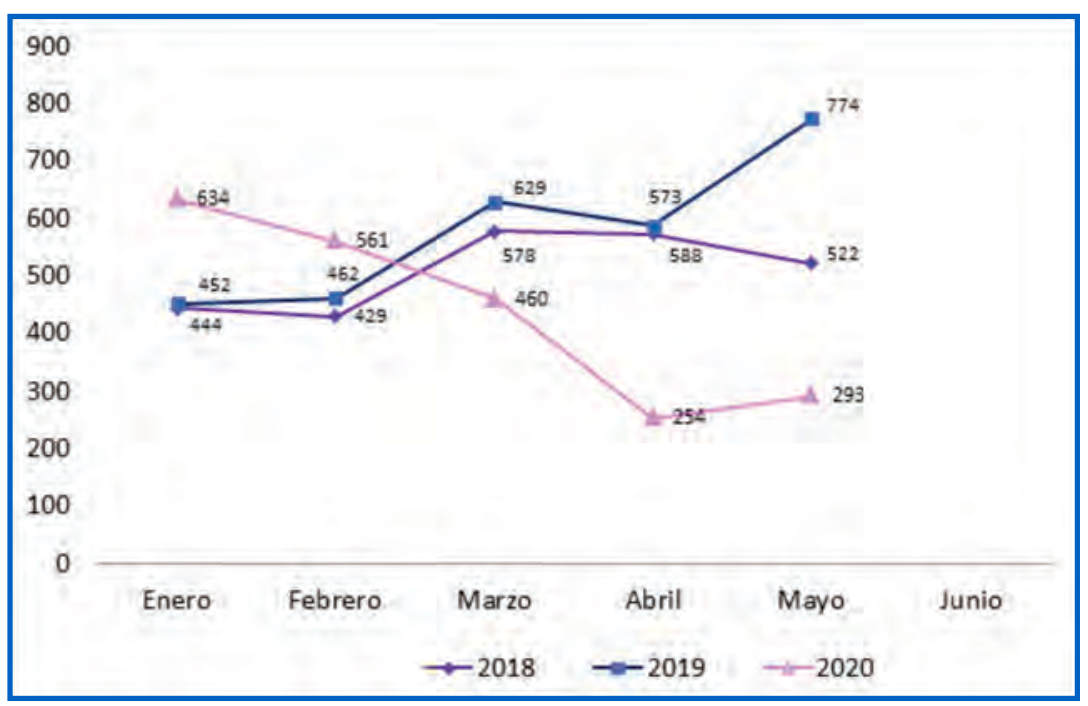

Fig. 2. Hospitalizaciones en el servicio de emergencias pediátricas, años 2018, 2019 y periodo pandemia hasta mayo 2020. Hospital General Pediátrico Niños de Acosta Ñu.

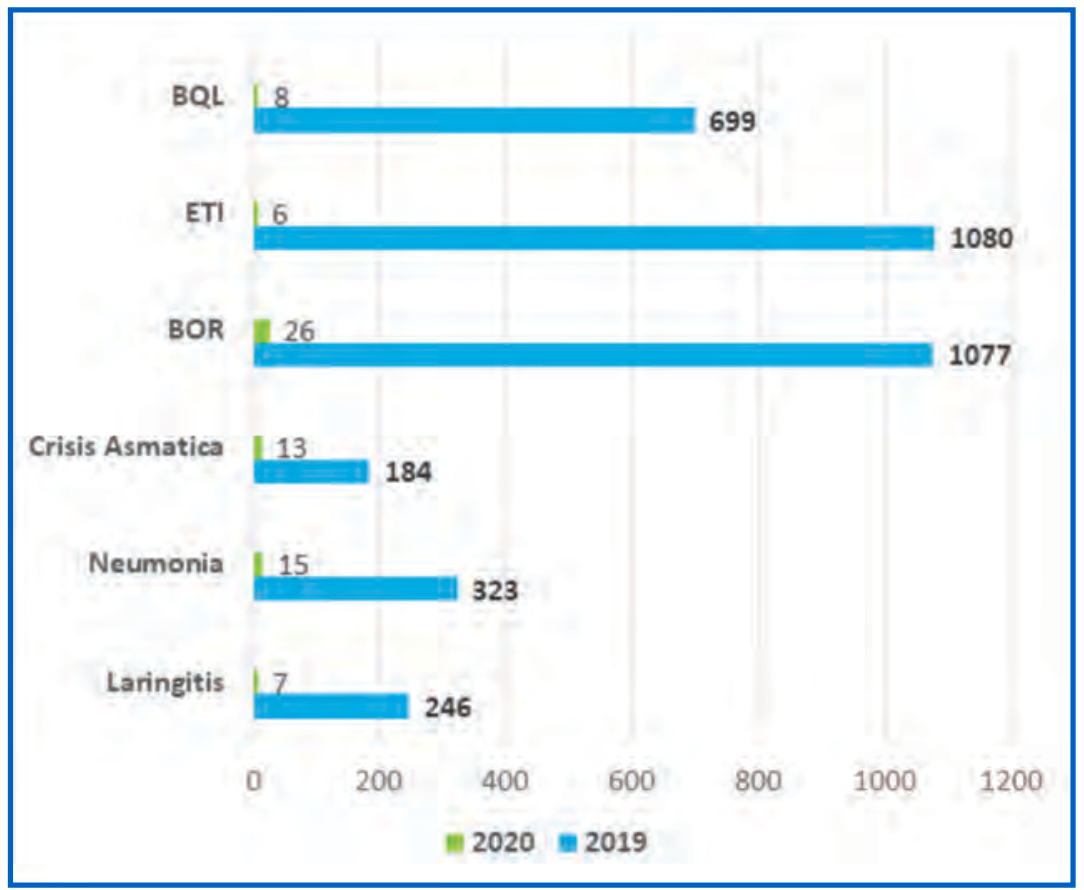

Fig. 3 Consultas por Enfermedades Respiratorias. Mayo 2019-2020.. Hospital General Pediátrico Niños de Acosta $\tilde{\mathrm{Nu}}$

$\mathbf{B Q L}=$ Bronquiolitis $\quad \mathbf{E T I}=$ enfermedad tipo influenza $\quad \mathbf{B O R}=$ Bronquial obstructivo recurrente 


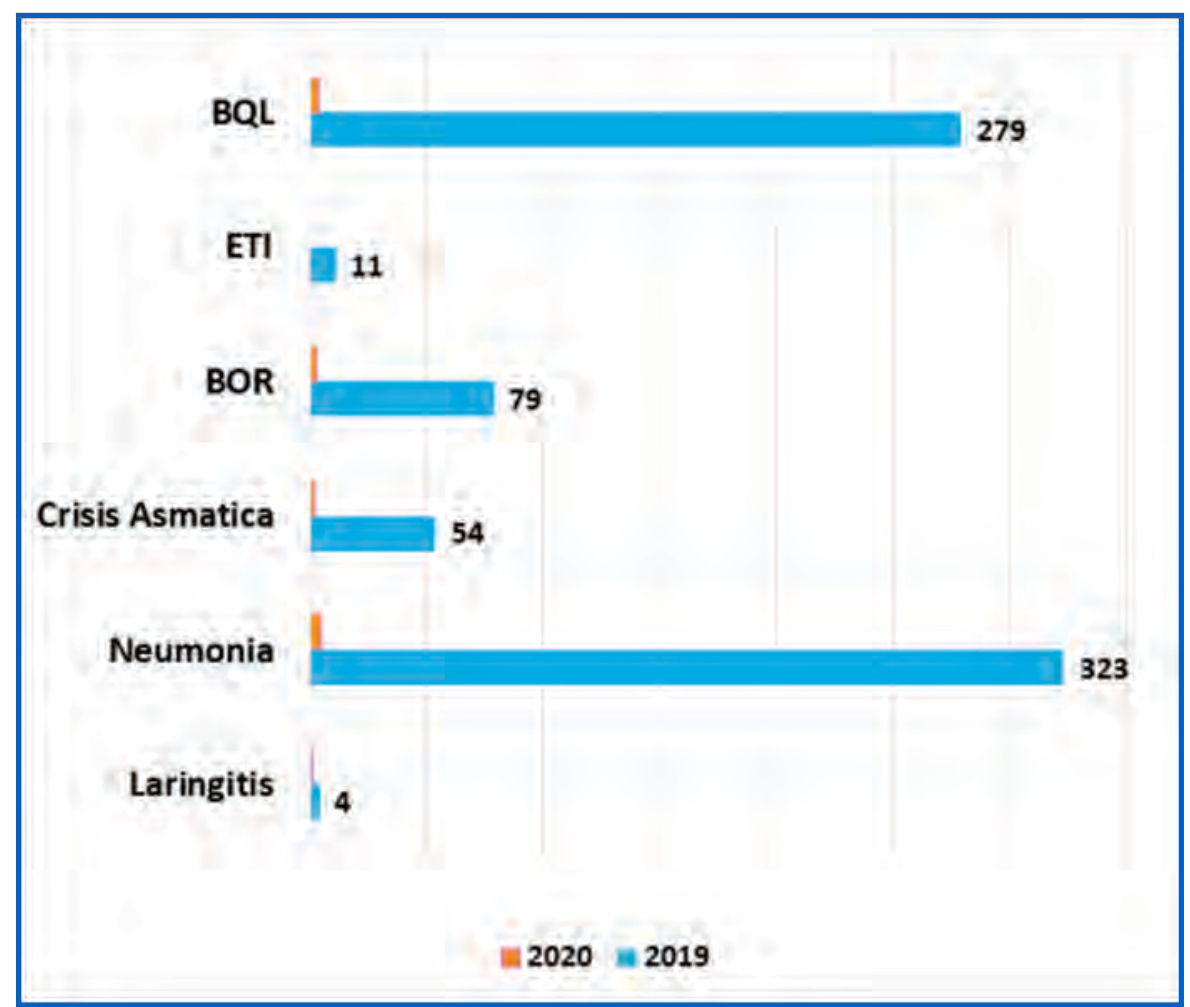

Fig. 4: Hospitalización por Enfermedades Respiratorias. Mayo 2019-2020.Hospital general Pediátrico Niños de Acosta Nu

$\mathbf{B Q L}=$ Bronquiolitis $\quad$ ETI=Enfermedad tipo influenza $\quad \mathbf{B O R}=$ Bronquial obstructivo recurrente 